CASO CLÍNICO

\title{
Colecistitis xantogranulomatosa, un reto diagnóstico para el radiólogo
}

\section{Xanthogranulomatous cholecystitis, a diagnostic challenge for the radiologist}

Emmanuel F. Murguía-Lugo*, José P. Martínez-Rivera y Agustín Parra-Macías

Servicio de Radiología, Hospital General Dr. Miguel Silva, Morelia, Michoacán, México

\section{RESUMEN}

La colecistitis xantogranulomatosa (inflamación fibroxantogranulomatosa de la vesícula) fue descrita por primera vez en 1970. Tiene una baja incidencia, del 0.7 al $10 \%$, y es más común entre la sexta y la séptima décadas de la vida. Se debe a una inflamación crónica de la vesícula por litos que condicionan la formación de nódulos xantogranulomatosos. Tiene un aspecto por imagen muy similar al carcinoma de vesícula, y de ahí la importancia de poder diferenciarlos. Presentamos un caso de nuestro hospital en el cual el diagnóstico preoperatorio fue de carcinoma de vesícula.

Palabras clave: Colecistitis xantogranulomatosa. Carcinoma de vesícula. Colecistitis crónica. Nódulos murales.

\section{ABSTRACT}

Xanthogranolumatous cholecystitis (fibroxantogranulomatous inflammation of the gallbladder) was first described in 1970. The incidence is low, between $0.7 \%$ to $10 \%$, and more common in the $6^{\text {th }}$ and $7^{\text {th }}$ decade of life. Occurs when a chronic inflammation of the gallbladder by stones contribute the formation of xantogranulomatous nodules. The image features can mimic gallbladder carcinoma, there is the importance about differentiate them. we presented a case of our hospital in which the preoperatory diagnosis was gallbladder carcinoma.

Keywords: Xanthogranulomatous cholecystitis. Gallbladder carcinoma. Chronic cholecystitis. Wall nodules. 


\section{INTRODUCCIÓN}

La colecistitis xantogranulomatosa, también conocida como inflamación fibroxantogranulomatosa de la vesícula, granuloma ceroide o granuloma histiocítico de aspecto ceroide, es una enfermedad inflamatoria variante de la colecistitis crónica ${ }^{1}$. Fue descrita por primera vez en 1970 por Singh et al. quienes observaron que la pared de la vesícula inflamada contenía nódulos ceroides (similares a cera). Tiene una incidencia del $0.7-10 \%$ y es más frecuente en la sexta y la séptima décadas de la vida, con una relación hombre-mujer de 2:1 y una asociación importante con la litiasis vesicular, de hasta el $80 \%{ }^{2}$. Los principales síntomas son dolor en el hipocondrio derecho, náuseas, vómitos, fiebre, anorexia e ictericia obstructiva, e incluso puede crecer hasta ser una masa palpable 2 .

En cuanto a su fisiopatología, se debe a una infección crónica de la vesícula biliar, generalmente en un contexto de colelitiasis, en la cual se forman microabscesos murales que involucran a los senos de Rokitansky-Aschoff. Esto puede resultar de la obstrucción del flujo de salida de la bilis, provocando que se extravase hacia la pared de la vesícula y la ulcere. Aquí los histiocitos se acumulan y forman los nódulos xantogranulomatosos, así como una reacción fibrosa extensa ${ }^{3}$.

Dentro de las complicaciones que puede llegar a dar se encuentra la compresión extrínseca del conducto biliar, y la aparición de ictericia. También puede llegar a formar fistulas que principalmente se dirigen hacia el duodeno, el colon, el antro del estómago e incluso la pared abdominal anterior ${ }^{4}$. Se ha visto que, por lo general, los estudios de laboratorio y los marcadores tumorales (Ca 19-9 y CEA) no son de ayuda para poder diferenciarlo del carcinoma de vesícula ${ }^{5}$. Debido a la clínica del paciente, es común que el primer método diagnóstico por imagen sea el ultrasonido, en el que se pueden observar litiasis vesicular, engrosamiento focal o difuso de la pared, y nódulos o bandas hipoecoicas intramurales (más característico) ${ }^{6}$.

En la tomografía pueden encontrarse realce continuo de la superficie de la mucosa, nódulos o bandas hipodensas intramurales, y líquido pericolecístico; se pueden llegar a ver trayectos fistulosos, la pared engrosada de manera focal o difusa, e inflamación que puede extenderse hasta el hígado y así perder una interfase bien delimitada, lo que hace que imite al cáncer de vesícula. En ocasiones, la litiasis vesicular no puede ser visible debido a la composición de los litos, y se puede acompañar de linfadenopatía reactiva local ${ }^{7}$.

Hay hallazgos útiles para diferenciar la colecistitis xantogranulomatosa del carcinoma de vesícula: en la colecistitis xantogranulomatosa es más común que el engrosamiento sea difuso en lugar de focal, que tenga realce continuo de la superficie de la mucosa, los nódulos intramurales de baja atenuación, la ausencia de invasión hepática, y es raro que se observe dilatación biliar. La presencia de tres hallazgos tiene una sensibilidad del $83 \%$ al $100 \%$ y una especificidad del $91 \%$ para la diferenciación entre colecistitis xantogranulomatosa y carcinoma vesicular?.

Es frecuente que la colecistitis xantogranulomatosa se diagnostique de manera inadecuada, ya que algunos hallazgos por imagen se superponen con los del carcinoma vesicular. Las tasas 
de carcinoma coexistente varían del 2\% al 15\% en los pacientes con colecistitis xantogranulomatosa ${ }^{8}$. La importancia de un diagnóstico adecuado es muy importante, ya que el carcinoma de vesícula tiene una tasa de supervivencia a 5 años menor del $5 \%$. Se sabe que representa el $0.5 \%$ de las neoplasias malignas gastrointestinales en los Estados Unidos de América?.

\section{CASO CLÍNICO}

Varón de 48 años que acude al servicio de urgencias de nuestro hospital por presentar ictericia y dolor abdominal. Inicia su padecimiento 4 semanas atrás con dolor abdominal intenso de inicio súbito, localizado en el hipocondrio derecho y el epigastrio, sin irradiaciones, el cual cedía con antiinflamatorios no esteroideos y se exacerbaba con la ingesta de alimentos, acompañado de náusea, distensión abdominal y vómito. Se agrega ictericia hace 3 semanas, sin acolia. Se le toman laboratorios, los cuales reportan ligera disminución de la albúmina y de la globulina. La bilirrubina total es de $5.6 \mathrm{mg} / \mathrm{dl}$, la bilirrubina directa de $5.2 \mathrm{mg} / \mathrm{dl}$ y la bilirrubina indirecta de $0.4 \mathrm{mg} / \mathrm{dl}$. La fosfatasa alcalina es de 628 U/1. El resto no muestra alteraciones. Se le realiza un ultrasonido de primera instancia en el que se observa engrosamiento focal con nódulos hipoecogenicos de la pared, y litiasis vesicular crónica con datos de agudización y dilatación de la vía biliar intrahepática y extrahepática (Fig. 1). Posteriormente se le realiza una tomografía abdominal con medio de contraste intravenoso en la que se observa la dilatación de la vía biliar extrahepática, así como realce continuo de la superficie de la mucosa vesicular y presencia de nódulos intramurales hipodensos (Fig. 2).
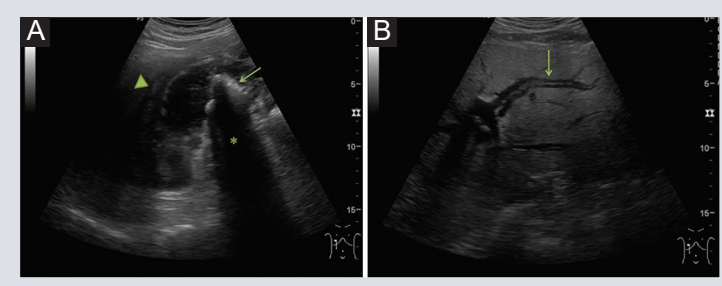

Figura 1. A: imagen por ultrasonido de la vesícula biliar que muestra los litos intraluminales (flecha), que proyectan sombra sónica posterior (asterisco), y el líquido perivesicular (punta de flecha). B: imagen por ultrasonido del lóbulo hepático izquierdo, con el signo en doble riel (flecha) asociado con dilatación intrahepática de la vía biliar.

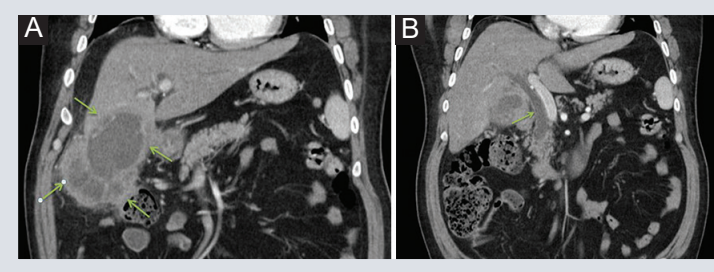

FiguRA 2. A: reconstrucción coronal de tomografía contrastada en la que se observa la dilatación del conducto biliar común (flecha). B: reconstrucción oblicua por tomografía contrastada de la vesícula biliar que muestra el reforzamiento continuo de la mucosa (flechas).

Se le realiza colecistectomía y en la biopsia transoperatoria se reporta colecistitis xantogranulomatosa (Fig. 3).

\section{DISCUSIÓN}

La colecistitis xantogranulomatosa es una rara condición inflamatoria de la colecistitis crónica, de baja incidencia y cuyas características pueden hacer que se confunda con un carcinoma de vesícula; por lo tanto, puede ser muy difícil distinguir prospectivamente entre una colecistitis xantogranulomatosa y un carcinoma de vesícula. Esto se debe a que muchas características se superponen, como el marcado engrosamiento de la vesícula biliar, 


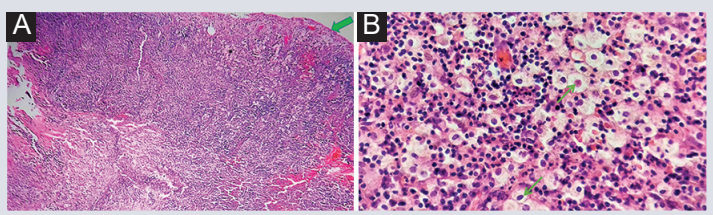

Figura 3. A: pared vesicular con infiltrado inflamatorio crónico grave a expensas de linfocitos y macrófagos espumosos (xantocitos) (asterisco). La mucosa vesicular se encuentra ulcerada, con proliferación vascular y fibrosis leve (flecha). B: macrófagos espumosos caracterizados como células grandes, poliédricas, de citoplasma amplio, claro y "espumoso", y núcleo blando central (flecha), entremezclados con linfocitos y polimorfonuclares.

la irregularidad y la infiltración de los tejidos blandos adyacentes. Si bien es una lesión benigna, puede tener un comportamiento agresivo por las características antes mencionadas. Al realizar la tomografía con medio de contraste intravenoso, en presencia de al menos tres datos tiene una sensibilidad del 83$100 \%$ y una especificidad del $91 \%$, por lo que si se pone atención a los hallazgos es posible hacer el diagnóstico diferencial.

El cáncer de vesícula es sumamente mortal, y de ahí la importancia de diagnosticarlo a tiempo para tener un mejor abordaje del paciente.

\section{FINANCIAMIENTO}

El presente caso clínico no presento financiamiento por parte de alguna institución publica o privada para su realización.

\section{CONFLICTO DE INTERESES}

Los autores declaran que no existen conflictos de intereses.

\section{RESPONSABILIDADES ÉTICAS}

Protección de personas y animales. Los autores declaran que para esta investigación no se han realizado experimentos en seres humanos ni en animales.

Confidencialidad de los datos. Los autores declaran que han seguido los protocolos de su centro de trabajo sobre la publicación de datos de pacientes.

\section{Derecho a la privacidad y consentimiento} informado. Los autores han obtenido el consentimiento informado de los pacientes y/o sujetos referidos en el artículo. Este documento obra en poder del autor de correspondencia.

\section{BIBLIOGRAFÍA}

1. Cárdenas-Lailson LE, Torres-Gómez B,Medina-SánchezS, Mijares-García JM, Hernández-Calleros J. Epidemiología de la colecistitis xantogranolumatosa. Cir Cir. 2005;73:19-22.

2. Singh VP, Rajesh S, Bihari C, Desai SN, Pargewar SS, Arora A. Xanthogranulomatous cholecystitis: what every radiologist should know. World J Radiol. 2016;8:183-91.

3. Bennett GL. Cholelithiasis, cholecystitis, choledocholithiasis, and hyperplastic cholecystoses. En: Gore RM, Levine MS, editores. Textbook of gastrointestinal radiology. $4^{\text {th }}$ ed. Philadelphia: Elsevier; 2015.p. 1348-91.

4. Zhao F, Lu PX, Yan SX, Wang GF, Yuan J, Zhang SZ, et al. CT and MR features of xanthogranulomatous cholecystitis: analysis of consecutive 49 cases. Eur J Radiol. 2013;82:1391-7.

5. Deng YL, Cheng NS, Zhang SJ, Ma WJ, Shrestha A, Li FY, et al. Xanthogranulomatous cholecystitis mimicking gallbladder carcinoma: an analysis of 42 cases. World J Gastroenterol. 2015;21:12653-9.

6. Parra JA, Acinas O, Bueno J, Güezmes A, Fernández MA, Fariñas MC. Xanthogranulomatous cholecystitis: clinical, sonographic, and CT findings in 26 patients. AJR Am J Roentgenol. 2000;174:979-83.

7. Federle MP, Raman SP, Borhani AA, Tublin M, Jeffey RB. Section 10 biliary system. En: Federle MP, Raman SP, editores. Diagnostic imaging: gastrointestinal. $3^{\text {rd }}$ ed. Philadelphia: Elsevier; 2015. p. 910-3.

8. Taskesen F, Arikanoglu Z, Uslukaya O, Oguz A, Aliosmanoglu I, Dusak A, et al. A rare finding during a common procedure: xanthogranulomatous cholecystitis. Int Surg. 2014;99:595-9.

9. Lee NK, Kim S, Kim DU, Seo HI, Kim HS, Jo HJ, et al. Diffusion-weighted magnetic resonance imaging for non-neoplastic conditions in the hepatobiliary and pancreatic regions: pearls and potential pitfalls in imaging interpretation. Abdom Imaging. 2014;40:643-62. 